\title{
Intracisternal Fibers in the Liver Cells of Starved Frogs
}

\author{
D. BAIC, B. E. Frye, and B. G. LADEWSKI \\ Department of Zoology, University of Michigan, Ann Arbor, Michigan 48104 \\ Received November 14, 1972
}

\begin{abstract}
Fibers observed within the cisternae of endoplasmic reticulum of liver cells of Rana pipiens are described. Fibers were observed only in animals subjected to starvation, but the frequency of fiber occurrence was found to vary widely from animal to animal and from cell to cell within the same animal. It is, therefore, suggested that starvation is important in, but not solely responsible for, the process of fiber formation. Some characteristic morphological changes observed in fiber-containing cells are described, and a tentative theory outlining a possible sequence of steps involved in fiber formation is presented.
\end{abstract}

It is well known that the rough endoplasmic reticulum has the capacity to accumulate and concentrate cell products within the lumen of its cisternae. Those cell products are rarely seen in electron micrographs because they are usually dissolved during tissue preparation for electron microscopy. However, numerous structures ranging from diffuse material through dense granules to highly organized tubular systems have been observed in endoplasmic reticulum. In some cells these are normal contents of the endoplasmic reticulum while in others they are a manifestation of a pathological process (1-7).

While studying ultrastructural changes in the liver of starved Rana pipiens we found numerous fibers in the endoplasmic reticulum of one frog. A careful reexamination of our material revealed that different amounts of fibers could be found in several more animals. This paper presents a summary of our initial findings. Further experiments are presently being undertaken in an attempt to isolate more frogs with fibers so that the chemical nature and significance of these structures can be determined.

FIG. 1. Portion of liver cell of Rana pipiens after 2 months' starvation. Two widened cisternae of rough endoplasmic reticulum containing fibers of different thickness. The wider fiber (arrow) shows discernible crossbanding. $\times 130000$.

FIG. 2. High power micrograph of a fiber exhibiting obvious crossbanding. $\times 200000$.

FIG. 3. Low power micrograph showing intracisternal fibers scattered throughout the cytoplasm. Only a few cisternae do not contain fibers. $\times 24000$.

FIG. 4. Three fibers within a single cisterna of rough endoplasmic reticulum. $\times 35000$. 

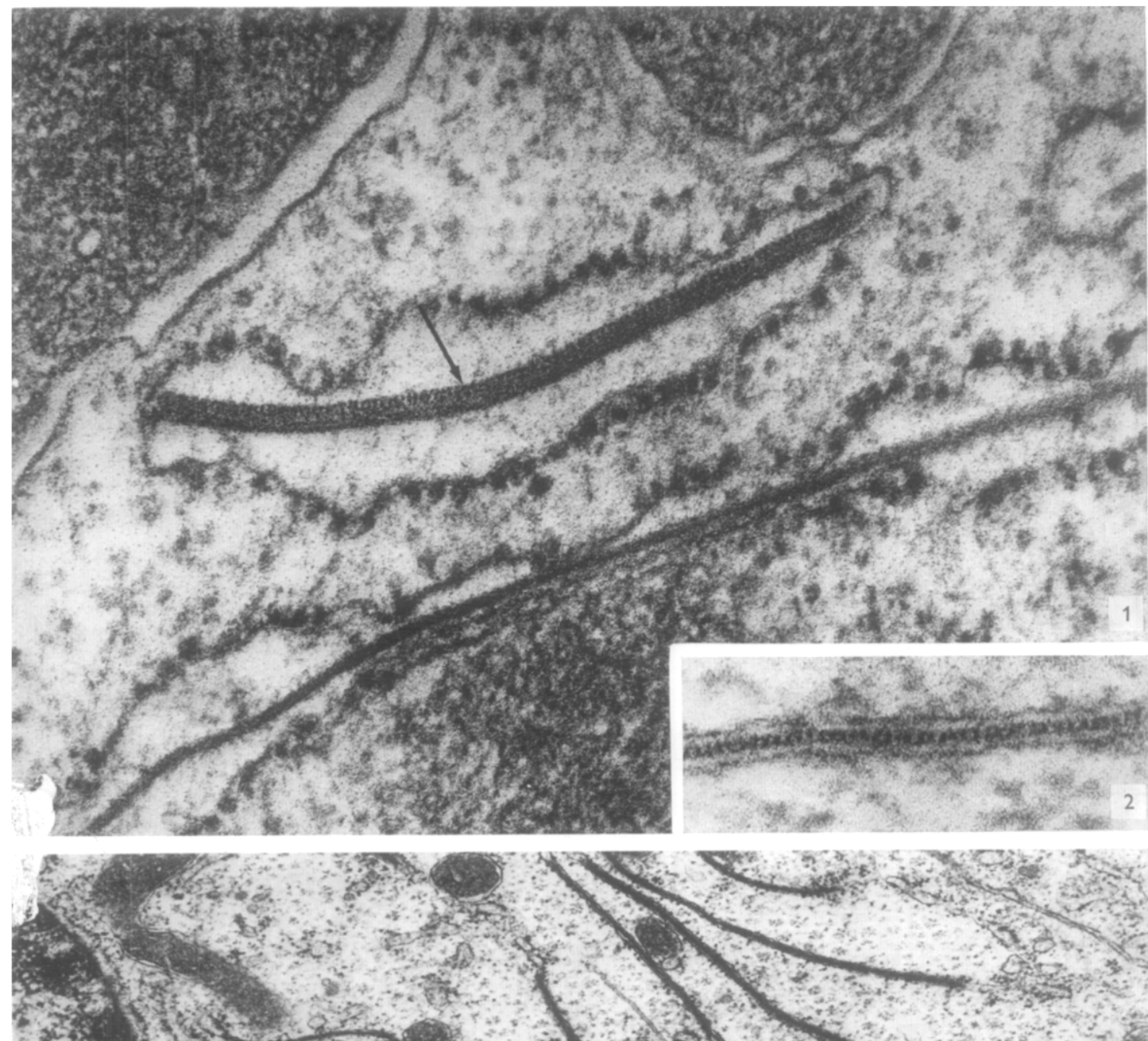

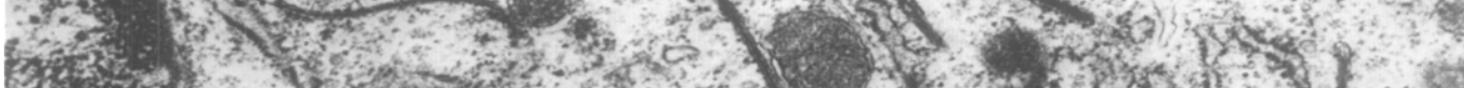

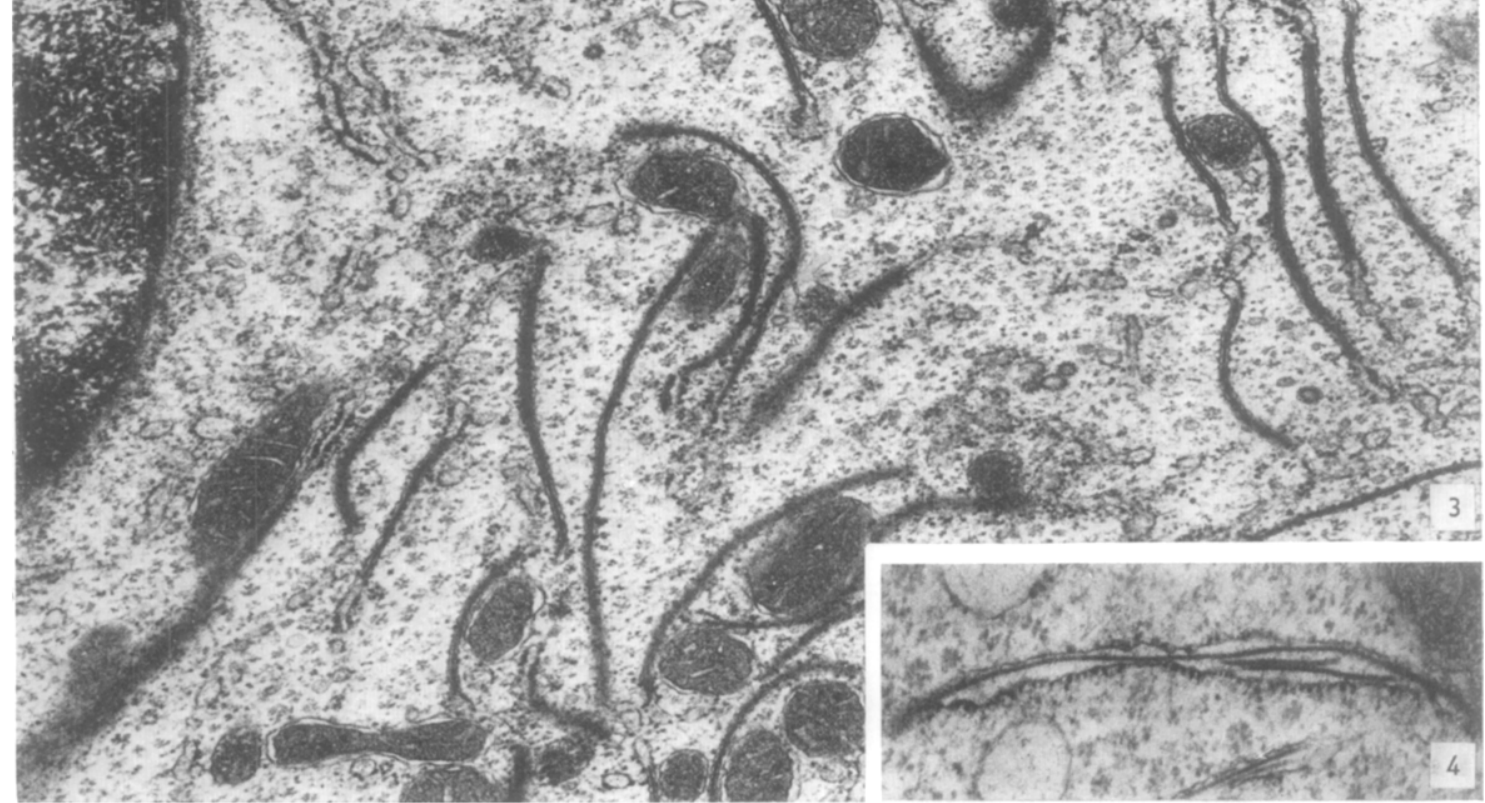


The tissue was fixed in glutaraldehyde, postfixed in osmium tetroxide, debydrated, and embedded in Araldite. The material was sectioned on a MT-2 ultramicrotome. The sections were contrasted with uranyl acetate and Reynolds' lead citrate and then examined with the Philips 300 electron microscope.

Figures 1, 3, and 4 show the appearance of fibers within the cisternae of the endoplasmic reticulum. As is evident in the micrographs, the fibers vary in length and thickness, as well as in density. Those in Fig. 3 range from 0.5 to $3 \mu \mathrm{m}$ long and are approximately 50-100 $\AA$ thick. At higher magnification (Fig. 2) the fibers show a banded pattern consisting of electron-dense subunits about $50 \AA$ wide separated by lighter regions approximately $75 \AA$ wide. As can be seen in Fig. 4, two or three fibers may be found parallel to each other within the same cisterna.

It should be noted that in almost all cases the fibers shown in these micrographs extend the full length of the cisternae of the endoplasmic reticulum, with no apparent breaks in their structure along this length. It should also be noted that the thickest fibers have indistinct outlines, frequently showing crossbanding only along one edge (Fig. 1, arrow). These observations have led us to the tentative conclusion that the "fibers" may actually be cross sections of beltlike bands within the lamellae of rough endoplasmic reticulum. This interpretation is supported by two additional observations: (a) serial sections in which the same "fiber" can be seen in as many as four consecutive sections, indicating a possible band width of up to $3000 \AA$; (b) apparent tangential sections (Fig. 5) in which the fibers appear as indistinct bands.

The possibility cannot be ruled out that the intracisternal bands observed in the rough endoplasmic reticulum occur also as true fibers within the tubular structure of the smooth endoplasmic reticulum. However, no cross sections of true fibers have been seen, even though many cross sections of tubular endoplasmic reticulum were observed in cells containing fibers.

There seems to be a definite transition in the development of the fibers within the cisternae of the endoplasmic reticulum. In early stages (Fig. 6) the constituents of the cisternae appear as an amorphous material which later becomes dense and forms the fiber with banded pattern already described. In advanced stages of development the fibers become increasingly electron-dense and the banded pattern is no longer distinguishable.

As the above fiber development occurs, the number of ribosomes on the affected cisternae decreases. In the final stages of fiber formation, often no ribosomes remain attached to the cisternae containing fibers (Fig. 3 and Fig. 6 at c).

FIG. 5. Portion of liver cell containing tangential sections of fibers (arrows). $\times 77000$.

FIG. 6. Slightly widened cisterna containing some amorphous material $(a)$. Adjacent is cisterna containing at point $b$ a more dense amorphous material and at point $c$ a crossbanded fiber. Note absence of ribosomes along cisterna where fiber is fully formed. $\times 110000$. 

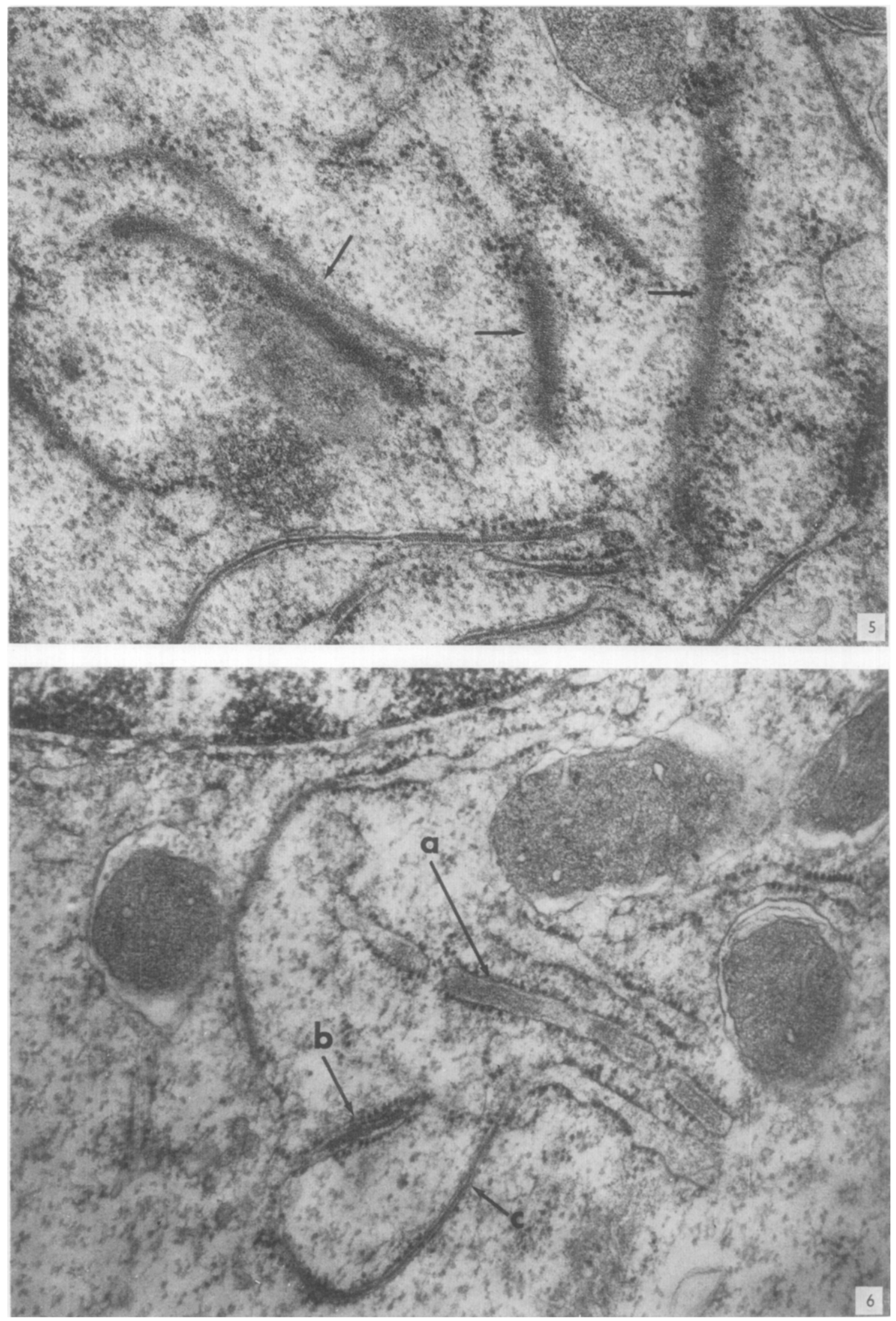
Other striking morphological changes appear to be associated with fiber formation in the cell. In cells having large numbers of fibers, the total amount of rough endoplasmic reticulum is greatly reduced; most of the remaining cisternae contain fibers and show the associated decrease in number of ribosomes as described above. In some such cells a decrease in the number of mitochondria and/or an increase in number of lipid droplets has been observed. Rosettes of glycogen which can be seen throughout the cytoplasm in the earlier stages of fiber formation, are greatly diminished in number or entirely missing in the later stages. No differences in nuclear appearance have been observed. It is important to note that the extent of the morphological changes seems to parallel the extent of fiber formation within the cell; little change in cell structure was observed in cells in the initial stages of fiber development.

Initial observations suggest that the occurrence of fibers within the endoplasmic reticulum of these cells may be correlated in some way with the process of starvation. However, since fibers do not occur with equal frequency in all the animals starved for identical periods of time, it is thought that another unknown factor or factors must also be involved.

This work was supported by faculty grant No. 354330 from the Horace H. Rackham School of Graduate Studies of the University of Michigan.

\section{REFERENCES}

1. Baringer, J. R. and Griffith, J. F., Science 165, 1381 (1969).

2. Chandra, S., Lab. Invest. 18, 442 (1968).

3. De MARTino, C., Z Zellforsch. Mikrosk. Anat. 97, 502 (1969).

4. FAWCETT, D. W., J. Nat. Cancer Inst. 15, Suppl., 1475 (1955).

5. Siekewitz, P. and Palade, G. E., J. Biophys. Biochem. Cytol. 4, 309 (1958).

6. Valeri, V., Gonçalves, R. P., Cruz, A. R. and Laicine, E. M., J. Ultrastruct. Res. 35, 197 (1971).

7. Weinstock, A., J. Histochem. Cytochem. 18, 875 (1970). 